\title{
INTERCAMBIO TRASFRONTERIZO DE SERVICIOS DE SALUD Y MEDICINAS EN LA REGIÓN DE TIJUANA Y SAN DIEGO
}

\author{
José G. Vargas-Hernández \\ jvargas2006@gmail.com \\ Departamento de Mercadotecnia y Negocios Internacionales \\ Centro Universitario de Ciencias Económico Administrativas \\ Universidad de Guadalajara \\ México
}

\section{RESUMEN}

Este trabajo tiene por objetivo analizar el intercambio fronterizo en la región Tijuana-San Diego de los servicios de atención médica, cuidados de la salud y medicamentos. Aun con un gran número de investigaciones y estudios, todavía se tienen muchos cuestionamientos con respecto al impacto de este intercambio en el desarrollo regional. El método empleado es exploratorio, analítico documental y de revisión de la literatura existente. En este trabajo se delimita el mercado trasfronterizo del sur de California y la zona fronteriza de Tijuana, las motivaciones de los usuarios y compradores, las principales barreras, características y tipología. Se enuncian algunas de las áreas para futuras investigaciones y finalmente se formulan algunas propuestas que tienen implicaciones en las políticas públicas. Este estudio arroja luz sobre la posibilidad de elevar los ingresos provenientes del comercio de los servicios de salud, mejorar la satisfacción de los usuarios y consumidores y mitigar las consecuencias negativas asociadas con el diseño de políticas y de iniciativas en los ámbitos multilateral, binacional, regional.

Palabras clave: atención médica, medicinas, mercado trasfronterizo, región Tijuana-San

Diego, servicios de cuidados de la salud. 


\section{ABSTRACT}

The objective of this article is to analyze the border exchange in the Tijuana-San Diego region of medical services, health care and medicines. Despite the numerous research studies conducted, there are still many questions regarding the impact from this exchange on regional development. The exploratory method, documentary analysis and a review of the literature were utilized. This article is focused on the transboundary market of southern California and the Tijuana border area, the motivations of users and buyers, the main barriers, characteristics and typology. Some areas for future studies are specified, and lastly, some proposals with implications for public policies are formulated. This study sheds light on the possibilities of increasing income from commerce in health services, improving the satisfaction of users and consumers, and mitigating the negative consequences associated with the design of policies and initiatives at the multilateral, binational and regional levels.

Key words: medical attention, medicine, transboundary market, Tijuana-San Diego region, health care services. 
ANTECEDENTES DEL INTERCAMBIO COMERCIAL DE SERVICIOS DE CUIDADOS DE LA SALUD, ATENCIÓN MÉDICA, MEDICINAS Y PRODUCTOS FARMACÉUTICOS

En las ultimas dos décadas, la globalización ha afectado todos los sectores productivos directa o indirectamente. Empujados en parte por los avances tecnológicos y por las convulsiones políticas y económicas, los procesos de globalización han dado lugar a la emergencia de nuevas formas de oportunidades, procesos y organizaciones de negocios. El sector salud ha sido afectado significativamente por la globalización económica a pesar de su naturaleza de bien público no comercial. El sector de servicios de cuidados de la salud está entre los sectores de más rápido crecimiento en la economía mundial, el cual se estima en alrededor de tres billones de dólares anuales solamente en los países de OCDE y alcanzó cuatro billones en 2005 .

La globalización de los servicios de la salud se refleja en la emergencia de nuevas formas de organización para los cuidados de la salud en la ultima década y en el incremento de la entrega trasfronteriza de servicios de salud a través del movimiento de productos y servicios. Una reestructuración mundial en el sector salud debido a los altos costos de los servicios médicos, particularmente en países desarrollados, ha resultado en la creación de un mercado internacional de cuidados y atención de la salud. El alto contenido de trabajo, capital y habilidades dentro de los servicios médicos provee una oportunidad para el desarrollo de los países, considerando que puede mantener los niveles de calidad necesarios. El comercio internacional en servicios de la salud en los países en desarrollo ha aparecido en la escena no solamente como una forma de incrementar sus ingresos sino también como una forma para fortalecer e incrementar los servicios nacionales de salud.

La compra de un servicio de salud en un país por parte de un consumidor que usualmente reside en otro país, considerado como el modo 2 de suministro internacional de servicios de salud de acuerdo con la Organización Mundial del Comercio, OMC, es un concepto que sirve de marco para la definición de turismo médico. No obstante, la definición es insuficiente para los fines de este trabajo, por lo que se considera para el comercio de 
servicios de cuidados de la salud la definición del comercio de servicios en general contenida en el artículo 2 del Acuerdo General sobre el Comercio de Servicio, que establece:

\footnotetext{
A los efectos del presente Acuerdo, se define el comercio de servicios como el suministro de un servicio: a) del territorio de un Miembro al territorio de cualquier otro Miembro; b) en el territorio de un Miembro a un consumidor de servicios de cualquier otro Miembro; c) por un proveedor de servicios de un Miembro mediante presencia comercial en el territorio de cualquier otro Miembro; d) por un proveedor de servicios de un Miembro mediante la presencia de personas físicas de un Miembro en el territorio de cualquier otro Miembro.
}

A las relaciones de intercambio comercial de servicios de cuidados de la salud y atención médica trasfronterizas también se las ha denominado intercambio de servicios de salud sin fronteras, lo cual implica la noción de pacientes sin fronteras.

\section{DELIMITACIÓN DEL MERCADO TRASFRONTERIZO EN LA REGIÓN SUR DE CALIFORNIA- NORTE DE MÉXICO}

La región fronteriza Tijuana-San Diego es un espacio geográfico que ha evolucionado como binacional, que coexiste con espacios económicos y sociales integrados y que se ha desarrollado en el trascurso del tiempo. Las personas que viven en las crecientes poblaciones de ambos lados de la frontera tienen cierto grado de interpenetración e intercambio que representan serios retos y oportunidades para la provisión de los servicios médicos y de salud. La frontera de California-Baja California es el hogar de $40 \%$ del total de la población que vive en la frontera entre México y Estados Unidos. Un intenso desarrollo urbano y un continuo flujo de personas y productos han trasformado las comunidades de esta frontera en una región distintiva con interacciones y relaciones sociales, económicas y políticas únicas.

Estas características tienen un impacto en el desarrollo físico y social. El ambiente político en la región de la frontera San Diego-Tijuana es a veces altamente contencioso. 
Diariamente hay una enorme «concentración» de transeúntes en el cruce de la frontera. Tijuana es el hogar de muchas familias binacionales, por el costo más bajo de vida y por la mezcla de la nacionalidad de sus familias, con miembros que trabajan en Estados Unidos. Junto a esta intensa migración diaria de sur a norte existe un flujo recíproco hacia el sur de nacionales mexicanos que viven al norte de la frontera y que viajan a Baja California por alimentos y servicios, así como también para mantener las ligas familiares, vecinales y de amistades.

La región Tijuana-San Diego cubre el noroeste de México y el sur de California, en donde hay desarrollo de telecomunicaciones, biotecnología, manufacturas, etc. La región fronteriza tiene uno de los mejores climas de Norteamérica. San Diego es famoso por sus playas fabulosas. La calidad de vida de San Diego es considerada de las mejores del mundo y de las más elevadas de Estados Unidos. Estos factores, aunados con los renombrados institutos de investigación y universidades, han llevado a compañías punta en biotecnología y telecomunicaciones a la decisión de instalarse en la región.

San Diego es un centro para la innovación de alta tecnología; se le conoce como Valle del Silicón de la biotecnología y las telecomunicaciones. La industria de defensa también juega un papel importante en la economía y la manufactura por su gran contribución al producto interno bruto regional. Por otro lado, Tijuana es uno de los centros manufactureros mundiales más competitivos para los negocios de mano de obra intensa, lo que ha atraído a muchas empresas europeas y asiáticas.

La región San Diego-Tijuana es la más grande de la frontera US-México, con una población combinada de más de cuatro millones de personas. El puerto de entrada a Estados Unidos, San Isidro, y Tijuana es el más transitado del mundo, con más de cincuenta y cinco millones de cruces en el año 2000 (COBBH 2000). El total de la población hispana de San Diego-Tijuana es 2,520, 835 de personas, y el total de hogares hispanos 610,720. El porcentaje de densidad hispana es 56.1\%, «casi» tres de cada cinco 
personas en la región es de origen hispano (San Diego Dialogue 1999, Tijuana DMA Estimates 2002). La importancia de la frontera US-México, con una población de más de diez millones, ha sido desestimada. Sharp (1998) la describe como un área de crecimiento sin prosperidad.

\section{EL MERCADO DE LOS SERVICIOS DE SALUD EN EL ÁREA TIJUANA-SAN DIEGO}

Se han realizado varios estudios (National Latino Research Center 2004) por diferentes organizaciones sobre la provisión de los servicios de salud en el área de la frontera entre Tijuana y San Diego. En 1963 se estableció en Tijuana la primera clínica con terapias no convencionales para tratar el cáncer a pacientes extranjeros (Moss 2005); a la que le siguieron otras, de tal forma que en 1968 Tijuana era ya un importante centro de tratamientos alternativos de enfermedades, que no era permitido en Estados Unidos (Smith 1968). Curiosamente, la literatura sobre el tema empieza a incrementarse en los años 90 debido al desarrollo basado en el incremento de la población hispana en los pueblos de la frontera y en la apertura del mercado mexicano a las inversiones como un resultado directo del TLCAN. Families USA (1992) reportó que miles de ciudadanos de Estados Unidos viajaban rutinariamente a pueblos de la frontera de México para atención médica.

Los residentes que viven en la frontera de Estados Unidos y México experimentan tasas más altas de enfermedades infecciosas, tales como la tuberculosis y las enfermedades prevenibles con vacunas, hepatitis, e infecciones intestinales por la falta de agua limpia y un adecuado deshecho de aguas negras. Subsecuentemente, los indicadores de salud y resultados en la región son igualmente de intensos y de integrados en ambos lados de la frontera. Los frecuentes movimientos entre ambos países, y dentro de Estados Unidos, comprometen la continuidad de los servicios de salud para los residentes de la región. Adicionalmente, la región presenta altas tasas de pobreza, desempleo y personas no aseguradas (National Latino Research Center 2004). 
Estos eventos han producido diferentes reacciones, que van desde estudios relacionados con el control y erradicación de enfermedades trasmisibles hasta estudios de mercadotecnia que buscan las oportunidades de inversión, sobretodo de Estados Unidos en el mercado de los servicios de la salud. Los estudios que existen sobre el comercio en la frontera no intentan buscar elementos de ventajas comparativas sino que proveen información que puede ser interpretada en ese sentido.

Sin embargo, se ha puesto poca atención del lado mexicano en el estudio del impacto que tiene el comercio de medicinas y de servicios de salud. Se han identificado 45 trabajos en la región de la frontera San Diego-Tijuana. Cuatro se efectuaron entre 1986 y 1989; 31 entre 1990 y 1996, y el resto posteriormente a estas fechas. La mayor parte de estos estudios corresponde a individuos y organizaciones de Estados Unidos, cuatro por instituciones mexicanas y solamente dos por investigadores de ambos lados de la frontera. La información proviene de seis grupos que han realizado las investigaciones.

El grupo que realiza estudios relacionados con el estado de la salud, el control y la erradicación de enfermedades transmisibles concluye en la necesidad de crear un programa binacional de atención a la salud. Otro grupo de estudios cubre temas de control migratorio con ciertas implicaciones xenofóbicas, a pesar de que son incapaces de demostrar que los indigentes mexicanos se convierten en una carga para el sector de salud pública de Estados Unidos. El tercer grupo de estudios se concentra en criticar los defectos y fallas del sistema de salud estadounidense. El cuarto estudia la tendencia de los estadounidenses para consumir medicinas y usar los servicios de salud mexicanos en la frontera, llegando a la conclusión de que lo hacen porque «todos los elementos necesarios están presentes». El quinto grupo toma en consideración las posibilidades de usar el sistema de salud pública de México como una posibilidad de reducción del gasto en salud de Estados Unidos.

Recientemente, después de la implementación del TLCAN, el sexto grupo realiza estudios de mercadotecnia sobre las oportunidades de Estados Unidos para invertir en el sector salud 
mexicano como una forma de captura del gasto que se realiza por los estadounidenses en México. A pesar de que casi todos los estudios reconocen que los estadounidenses viajan a México para atenderse en los servicios médicos, solamente algunos lo ven como una nueva tendencia. Los estudios que tratan de las oportunidades de inversión de los estadounidenses y la reducción del gasto en salud en Estados Unidos se enfocan hacia diferentes áreas, y desde diferentes perspectivas; estos esfuerzos se consideran formas para alentar el comercio internacional en los servicios médicos entre México y Estados Unidos.

El gasto en cuidados de salud representa más de $16 \%$ del producto interno bruto de Estados Unidos, el más alto en gasto per cápita en cuidados de salud entre los países desarrollados. Estados Unidos enfrenta una crisis de los servicios de salud por la falta de acceso, o limitado, de algunos grupos de población, los altos costos de los servicios de salud y medicinas, por falta de calidad en los servicios que se ejemplifica en el alto grado de errores médicos y de medicación que se documentan. Un estudio de California HealtCare Foundation (2001) reporta un incremento de $18.8 \%$ en tres años del consumo de productos farmacéuticos. La reimportación de medicinas y productos de este tipo aumenta en los últimos años ante la falta de monitoreo y administración de productos y cuidados al paciente.

A pesar de que introducir cualquier droga prescrita en territorio de Estados Unidos es técnicamente ilegal, la agencia Food and Drug Administration estima que más de dos millones de paquetes de productos regulados entran anualmente a través del correo. La proximidad de California con la frontera mexicana hace la salud binacional y fronteriza como sinónimos para muchos profesionales del sector salud. Las relaciones comerciales trasfronterizas en la región son muy intensas tanto entre consumidores de servicios de salud y proveedores en forma presencial así como en el tráfico electrónico y por otros medios. Esto se refleja en el creciente número de empresas y organizaciones involucradas en el sector de los servicios de salud, que tienen acuerdos de colaboración, alianzas estratégicas, 
joint ventures, etc.; de igual manera se refleja en la intensificación de los intercambios fronterizos para la diseminación de información, educación y entrenamiento en este sector.

En los años recientes ha habido un crecimiento significativo en oportunidades y en las formas de comercio e inversión directa extranjera, IED, entre los dos países, uno desarrollado y otro en desarrollo. Las implicaciones de la presencia comercial también puede generar recursos adicionales para la inversión en la mejora de la infraestructura de los servicios de salud y las tecnologías, de la misma forma generar empleo y reducir el desempleo del personal de salud. Puede asimismo permitir la provisión de servicios médicos costosos y especializados e incrementar la competitividad, capacidad, calidad, accesibilidad y productividad de los servicios de salud.

Considerado uno de los más desarrollados mercados latinoamericanos de farmacéuticos, la infraestructura regulatoria de México promueve el crecimiento de la manufactura farmacéutica. Más de 170 plantas de manufactura operan cerca de 400 instalaciones y se dan facilidades a empresas de prestigio: Pfizer, Janssen-Cilag, GlaxoSmithKline, ScheringPlough, y Bristol-Myers Squibb, entre otras. De estas, algunas se encuentran en Tijuana. La disponibilidad del capital privado puede reducir la carga total de los recursos del gobierno y ayudar a reasignar el gasto gubernamental hacia el sector público del cuidado y atención de la salud. Hasta ahora el gobierno no ha desempeñado un papel importante en el desarrollo del comercio internacional en el sector de los servicios médicos. Por lo tanto, los temas que están relacionados con la intervención del gobierno son los que se relacionan con la telemedicina, una forma que el gobierno mexicano ha promovido para la entrega de servicios médicos a las áreas remotas, proponiendo facilidades e instalaciones en los cruces fronterizos; una política que el gobierno mexicano considera importante debido al influjo de los turistas.

Además crea oportunidades para los entrenados de los países en desarrollo para buscar entrenamiento en centros internacionales de excelencia. La presencia comercial puede 
también hacer posible la mejora de su calidad a través de la introducción de técnicas de administraron superior y de sistemas de información. También puede haber externalidades positivas de entrenamiento nacional para las instituciones. La presencia comercial foránea en el sector de la educación médica en la forma de joint ventures entre escuelas de medicina locales y extranjeras puede ayudar a los países receptores a diferenciar y a mejorar la currícula de estudios mientras se generan ingresos para la institución exportadora y se alienta la reputación técnica y científica.

Lo más importante, para nuestra investigación es que TLCAN ha dado un impulso a la movilidad trasfronteriza de consumidores dentro de la región. Clínicas privadas de Tijuana están cubriendo parte del mercado de servicios de salud de las ciudades fronterizas de San Diego con base en servicios de alta calidad y precios bajos. Con el avance en la integración de los sistemas de salud, las compañías de seguros, HMO, y clínicas pueden conjuntamente proveer servicios médicos a consumidores estadounidenses a precios muy bajos. Debe ser notado, sin embargo, que incluso fuera del TLCAN, siempre ha habido un movimiento trasfronterizo de pacientes entre Estados Unidos y México.

El movimiento trasfronterizo de servicios siempre ha existido como la trasmisión de información de diagnóstico o terapéutica, mediante la comunicación normal nunca ha sido restrictiva. En todo caso, por varios años un número de laboratorios privados mexicanos han preguntado regularmente a Estados Unidos por ciertos tipos de exámenes especializados de laboratorio. Similarmente, los impactos crecientes de la salud trasfronteriza están logrando ser más pronunciados.

En promedio mensual había en 1994 más de 300,000 cruces de frontera de Estados Unidos a México, y viceversa, para el consumo de servicios médicos, de los cuales 50,000 fueron desde los pueblos fronterizos de México a San Diego y el resto desde los pueblos fronterizos de Estados Unidos a Tijuana (UNCTAD/WHO 1998). Los resultados de la encuesta realizada por la Universidad de California en Los Ángeles (UCLA 2001) 
mostraron que en un año viajaron a México 1,060,000 californianos para procurarse atención médica, cuidados dentales, medicamentos y otros servicios de cuidados de la salud. No obstante, otra encuesta realizada en 2003 demostró que los ciudadanos de ambos lados, San Diego y Baja California, llevan a cabo al menos 100,000 cruces mensuales de frontera relacionados con la atención de servicios médicos (Terman 2004).

Según las investigaciones de Olivieri (2007) alrededor de 40\% de los extranjeros que visitan la región fronteriza mexicana por motivos de atención y cuidados a la salud se regresa el mismo día. Se estima que los médicos privados de Tijuana atienden diariamente a 12,000 pacientes méxico-americanos y estadounidenses, y solamente a 3,000 nacionales (Frontera 13 de junio de 2006). Con datos más recientes, La metrópolis de Tijuana recibe 250,000 visitantes mensualmente para comprar sus medicinas prescritas o sobre el mostrador y recibir cuidados y atenciones médicas y dentales. Los aproximadamente 250,000 cruces mensuales de frontera realizados por los californianos para recibir atención médica, servicios de cuidados de salud y adquisición de productos farmacéuticos, crean un mercado de 500,000,000 de dólares anuales (Osio 2010).

De acuerdo con datos aportados por la Secretaría de Turismo y Comité de Turismo y Convenciones de Tijuana 2003, en este año Baja California obtuvo \$18,000,000 de dólares por concepto de provisión de servicios médicos y atención de cuidados de la salud a extranjeros. Por otra parte, el gobierno del estado de Baja California estimó que la atención médica y cuidados de la salud a los usuarios y sus acompañantes dejaban \$2,000,000 de dólares al año, mayor a lo que obtuvo India por el mismo concepto (El Mexicano 11 de junio de 2007, Frontera, 17 de junio de 2007). Se estima que más de \$1,300,000 de dólares se gastan anualmente en servicios de salud en el condado de San Diego.

En promedio se estipula que cada ciudadano de San Diego gastó \$3,759.224 en 2001, comparativamente uno de los más altos de Estados Unidos, a pesar de que todavía $21 \%$ del 
segmento de la población no anciana (edades 0-64) no tienen o solo parcialmente cuentan con seguro médico (Brown et al. 2003: 23).

\section{LAS MOTIVACIONES DE LOS USUARIOS DE LOS SERVICIOS DE ATENCIÓN Y CUIDADOS DE LA SALUD}

Entre las motivaciones de los usuarios que explican la alta demanda de los servicios médicos y de salud en la región fronteriza de Tijuana se mencionan los precios más bajos que en el mercado de Estados Unidos, la carencia de seguro médico (Escobedo y Cárdenas 2006, Macías y Morales 2001) y la afinidad cultural de las relaciones con el paciente. Otra motivación importante se sustenta en las diferencias regulatorias y de supervisión para el acceso a los servicios de atención y cuidados de la salud y la adquisición de medicinas. La American Medical Association (2007) señala también como motivaciones importantes la mayor especialización y calidad alcanzada en el extranjero, y más facilidades en los viajes internacionales. Hay que considerar además como motivación la expedición de la nueva forma de seguros trasfronterizos por empresas aseguradoras trasfronterizas.

\section{PRECIOS MÁS BAJOS}

El alto costo de los servicios de atención médica y cuidados de la salud en Estados Unidos da lugar a que los estadounidenses busquen alternativas, entre ellas da lugar a que un creciente número de personas crucen la frontera para obtener mejores beneficios en sus compras en Tijuana y en la región fronteriza. Los servicios de atención médica y cuidados de salud, así como los medicamentos y otros productos farmacéuticos, son más accesibles en precio en Tijuana que en San Diego, y por ende en California, porque se ofrece a través de agencias públicas que reciben subsidios, pero sobretodo a factores tales como los bajos costos laborales, poco trabajo administrativo y medicina más barata.

Con respecto a los precios más bajos, el costo de medicinas y productos fármacos en México son alrededor de 30\% más baratos que en Estados Unidos. De acuerdo con datos proporcionados por el Colegio de Médicos Generales (Frontera 13 de junio de 2006), los 
pacientes que viajan a Tijuana se ahorran de $30 \%$ a $50 \%$ en sus consultas clínicas y de $30 \%$ a $80 \%$ en tratamientos quirúrgicos, equivalente a ahorros que van de \$20 a \$50 dólares en casos de una consulta, y de $\$ 3,000$ a $\$ 20,000$ dólares, o mucho más, en casos de intervenciones quirúrgicas.

Por ejemplo, con datos de US Senate Special Commite on Aging (2006) el costo de una cirugía mayor aortocoronaria o CABG en el Hospital Ángeles de la ciudad de México es de $\$ 25,000$ dólares, que presumiblemente sería menor en cualquier hospital de Tijuana pero que en todo caso representa menos de la mitad de $\$ 60,400$ dólares que cobran en promedio los hospitales estadounidenses.

A pesar de la amplia idea de que los servicios médicos de México son de muy baja calidad, en general los estadounidenses viajan al sur de la frontera buscando servicios de salud asequibles y medicinas de bajo costo, encontrando además que los niveles de la calidad son también aceptables. Lo anterior se confirma con los siguientes datos reportados por la encuesta de UCLA (2001), donde 71\% de los 651,000 californianos que viajaron a México para comprar medicinas no visitaron médicos ni consultaron dentistas mexicanos. Se estimó en 253,000 los compradores estadounidenses de medicina en México (UCLA 2001).

\section{CARENCIA DE SEGURO MÉDICO}

En California, un tercio de los hogares no tienen cobertura de seguro de salud o presentan cobertura limitada, incluyendo a una mayoría de hogares latinos (Osio 2010). En California, más de $70 \%$ de los trabajadores migrantes del campo y sus familias carecen de cobertura de seguro médico (International Community Foundation 2008). Mas de 34,000,000 de residentes estadounidenses no asegurados requieren de un sistema de cuidados de salud más accesible y económicamente disponible. De acuerdo con California HealthCare Foundation, una organización sin fines de lucro con sede en Oakland, los latinos en California representaron 59\% de 6,600,000 de no asegurados en California en 2006 (Darcé 2008). 
Para el año 2003, según Robert Wood Johnson Foundation (2005), carece de seguro médico 18.9\%, que representa más de 4,000,000 de la población adulta entre 18 y 64 años, de los cuales 2,298,628, más de la mitad, son de origen latino, y 2,371,570 de los no asegurados trabajan. Trabajan pero no cuentan con seguro médico, 15.9\%. Para el mismo año, 1,625,002 adultos sin seguro no tuvieron para pagar la atención médica.

Como se comentó arriba, el estado de California tiene una población de 35,000,000 de habitantes, de los cuales 6,300,000 de residentes no tienen seguro De estos, 3,300,000 de personas han estado sin seguro por más de un año (Brown et al. 2003: 11-12). Los californianos de bajos ingresos son más propensos a estar sin seguro por un tiempo más largo.

Un promedio de $86 \%$ de los infantes en edades entre 0 y 17 tienen seguro médico. Entre los niños pobres de familias con ingresos menores a 100\% del Nivel Federal de Pobreza (Federal Poverty Level, FPL), el $14.4 \%$ no tiene seguro y otro $10.4 \%$ solamente cubre una parte del año (Brown et. al. 2003: 18). Uno de cada cuatro de los niños californianos pobres ha carecido de cobertura de seguro médico continuamente. Los niños de hogares pobres o casi pobres — abajo de $200 \%$ de FLP — son $80 \%$ de los niños sin seguro médico (Brown et al. 2003: 17). La cobertura del seguro médico es más baja para la población adulta (edades entre 18 y 64). Solamente un poco más de $50 \%$ de los adultos en pobreza tienen seguro todo el año, comparado con $88 \%$ de aquellos hogares que ganan $300 \%$ o más de FLP (Brown et al. 2003: 16).

La cobertura de salud varia de acuerdo con el ingreso, la etnicidad y el estatus de ciudadanía e inmigración. De los latinos $28 \%$ en edades entre 0 y 64 años no están asegurados, comparados con solamente 9\% de los blancos (Aguayo et al. 2003: 1). Los niños latinos son cinco veces más propensos a no tener seguro de salud que los blancos o africanos. De los niños americanos, más de $90 \%$ de blancos, afroamericanos y asiáticos 
americanos/isleños del Pacifico, están asegurados todo el año, comparado con solamente 76\% de los niños latinos (Brown et al. 2003: 18). Los adultos latinos menores de 65 años tienen una tasa de $28.5 \%$, la más alta de no asegurados, durante todo el año. Son los «no» ciudadanos los que más carecen de seguro médico: $44 \%$ de adultos y $34 \%$ de niños no tienen seguro (Brown et al. 2003: 19-20).

Los latinos tienen menor cobertura de seguro médico porque no pueden pagar un seguro, otros son ilegales y no pueden comprar un seguro, porque no son elegibles para Medicare o Medical, o no tienen suficiente recursos para contratar una alternativa en la consulta privada (Guendelman y Wagner 2000). De la población adulta, 29\% de latinos, 12\% de blancos y $12 \%$ de afroamericanos. Los empleadores no pagan seguro a 57\% de los latinos no ancianos, en contraste con $24 \%$ de los blancos no ancianos. En estos datos las diferencias muestran desventajas para los latinos, principalmente porque se emplean en pequeños negocios y empresas que no están obligadas a proporcionar seguro médico a sus trabajadores.

De acuerdo con Valdez et al. (1993: 889), los hispanos tienen la peor cobertura de seguro de salud, en cuanto a grupo étnico, en Estados Unidos. De los hispanos, 39\% no está asegurado, comparado con $13.8 \%$ de los anglos y $24 \%$ de la población negra. Al menos la mitad de los que no tienen seguro en California, entre 6,200,000 y 6,400,000 de personas sin seguro de salud de ninguna clase son hispanos. El gran número de personas que no tiene acceso a programas de seguridad con cobertura para los servicios médicos y de salud se estima que oscila entre 645,000 en San Diego y 565,000 en Tijuana (Warner s.f.).

Desde este lado de la frontera, un reporte preliminar de investigación sobre las relaciones trasfronterizas y cuidados de la salud en Tijuana realizado por Wallace y Ybanez (2006), con información colectada en 264 hogares, encontró que 29\% de las personas no cuenta con seguro médico y $60 \%$ tiene la cobertura del Instituto Mexicano del Seguro Social. El Censo de Población del año 2000 en Tijuana muestra que 47\% de los residentes tiene cobertura de 
seguros médicos y de salud y la mayoría, más de 90\%, a través del programa de atención médica pública, es decir, a través del Instituto Mexicano del Seguro Social o del Instituto de Seguridad y Servicios Sociales de los Trabajadores al Servicio del Estado (International Community Foundation 2008b, con datos de Lomelí 2001: 2).

Quienes carecen de seguro médico no consiguen un cuidado de la salud adecuado, por lo que se exacerban las enfermedades o heridas que con frecuencia requieren de servicios de emergencia con cuidados y atenciones médicas más caros. Esta situación se considera como resultado de un modelo de provisión de cuidados de la salud y atención médica que es ineficiente y costoso.

Los no asegurados no son siempre los pobres. Muchos trabajadores de la clase media tampoco pueden pagar las pólizas crecientes de los seguros médicos, lo que los hace prohibitivos para las empresas pequeñas y medianas que ofrecen seguro de salud como un beneficio a sus empleados.

\section{AFINIDAD CULTURAL EN LAS RELACIONES PACIENTE-PROVEEDOR DE SERVICIOS DE ATENCIÓN Y CUIDADOS DE LA SALUD}

Muchos californianos cruzan la frontera con México para entrar en Tijuana en búsqueda de atención médica, cuidados de salud y medicinas que sean no solamente accesibles en precios sino también culturalmente competentes y afines. Si bien muchos latinos tienen mayores posibilidades de acceso a los sistemas de provisión de cuidados de la salud y atención médica en ambos lados de la frontera, acuden con mayor frecuencia a Tijuana por la necesidad de sentirse culturalmente más competentes y afines porque se sienten más confortables y más familiarizados que con la cultura de atención médica de Estados Unidos debido a las barreras del idioma y a las diferencias culturales.

Es en este contexto que el concepto de interculturalidad en salud toma mayor relevancia porque vincula las identidades culturales de seres humanos frente a otros seres con su 
propia identidad cultural, valores, creencias, visiones, tradiciones, costumbres que pueden entrar en contrapunto con las prácticas hegemónicas de la medicina tradicional.

La afinidad cultural y de idioma son factores cruciales para la buena comunicación entre el agente proveedor de los servicios de atención y cuidado de la salud y el paciente, para recibir y seguir las indicaciones de los tratamientos médicos. Doheny K. (2006) puntualiza que «Los pacientes que no tienen la oportunidad de tener un médico competente cultural y lingüísticamente con frecuencia no obtienen una atención tan buena. Poder hablar con el paciente es un asunto crítico». Para Villa-Caballero, Caballero-Solano y Andrade-Barreto (2008) la determinación del nivel de culturización y tendencias en usos y costumbres de la población que vive en la frontera con respecto al estilo de vida norteamericano es un reto para la investigación futura.

Una investigación reportada por International Community Foundation (2008b) encontró que los trabajadores del campo en Estados Unidos prefieren la atención médica y el cuidado de su salud en México porque esperan un acercamiento al tratamiento que implica el uso de medicina potentes y que actúan rápido, pocas pruebas de laboratorio y mínimos trámites burocrático-administrativos, y sobretodo para quienes no hablan inglés y tienen problemas con la comunicación, además de que las presiones por la demanda de tiempo y recursos son altas. Para los pacientes de origen mexicano y latino, el sistema de salud del lado de Tijuana es culturalmente competente, expedito y de bajo costo.

DIFERENCIAS REGULATORIAS Y DE SUPERVISIÓN DE LA CALIDAD DE LOS PRODUCTOS FARMACÉUTICOS Y LOS SERVICIOS DE SALUD

A pesar de que el mercado de prescripción de medicamentos en Baja California es más eficiente que en San Diego, la regulación es menos rigurosa. Las diferencias regulatorias y de supervisión de la calidad de los tratamientos, servicios y fármacos para la atención y cuidados de la salud son abismales entre los dos países, siendo más permisiva la mexicana y más estricta la estadounidense. La disponibilidad y acceso a productos farmacéuticos y me- 
dicinas es «over the counter» en Tijuana sin que se requiera receta médica, mientras que para algunos medicamentos tienen un control más estricto en Estados Unidos donde solo se venden bajo estricta prescripción médica, de acuerdo con los estándares de Food and Drugs Administration, FDA.

Esta desigualdad regulatoria ha dado lugar a una proliferación de «clínicas» que ofrecen tratamientos alternativos para enfermedades degenerativas y crónicas, tales como el cáncer, que no están permitidas en Estados Unidos (American Cancer Society 1991-2002). La investigación de Moss (2005) reporta más de 25 clínicas ubicadas principalmente en el centro y zona río de Tijuana, Playas de Tijuana, La Meza y Rosarito. La regulación y supervisión de las normas mexicanas de salud, de atención médica, de medicinas y productos farmacéuticos, enfrentan el reto de tener un control en la venta y mejorar la calidad de medicinas.

Con respecto al sector farmacéutico, la liberalización controlada puede dar a oportunidades a la competencia. Sin embargo, desde que la medicina privada se expande poco a poco y limitadamente a las zonas urbanas, y la pobreza absoluta es todavía frecuente y común, la no proliferación de productos farmacéuticos puede esperarse razonablemente. Para la introducción de nuevas drogas en el mercado, se requiere más información relacionada con su utilización y efectos secundarios para quienes prescriben y para los pacientes. No obstante, el peligro es que estas medicinas consuman una parte no razonable de los recursos disponibles.

NIVEL DE ESPECIALIZACIÓN Y CALIDAD DE LOS SERVICIOS DE ATENCIÓN Y CUIDADO DE LA SALUD

La colegiación médica, la certificación y recertificación de los médicos no es obligatoria, y mucho depende de la voluntad del médico, a diferencia de Estados Unidos donde es un requisito exigible para la práctica médica. Menos de 50\% de los médicos especialistas son miembros o están afiliados a sus respectivos colegios médicos. El Consejo de Desarrollo 
Económico de Tijuana, apoyado por el gobierno de Baja California norte, coordina la asociación de prestadores de servicios, misma que integra un clúster de servicios de salud con funciones de organización y representación de intereses además de recomendar y promover la certificación de médicos y hospitales para reducir los riesgos a la salud de los pacientes (Ramírez 2008).

\section{PLANES DE SEGUROS TRASFRONTERIZOS}

Los seguros médicos trasfronterizos tienen como ventaja que toman en cuenta las fortalezas de los dos sistemas de salud en ambos lados de la frontera. Las necesidades de atención médica que enfrenta la industria de cuidados de la salud son tan grandes que ya existen proveedores que ofrecen programas alternativos a los usuarios sin seguros médicos, para beneficios limitados a través de los empleadores que facilitan el acceso a los servicios de salud. Los planes trasfronterizos de seguros portables que ofrecen algunas compañías de seguros médicos dan atención médica a sus derechohabientes en el extranjero.

Los empleadores de California que ofrecen planes de salud de seguros trasfronterizos están aumentando (Anfuso 1995). Los planes de seguros para los trabajadores mexicanos en los condados fronterizos de San Diego e Imperial se han legalizado desde el año 2000, y más de 160,000 trabajadores recibían los beneficios en 2005 (Los Angeles Times 25 de agosto de 2005, San Diego Union Tribune 16 de octubre de 2005).

Una parte son indocumentados que ahora sí pueden contratar servicios de salud para ellos y sus familias con la aceptación de la Matrícula por parte de Blue Cross Blue Shield of California, Health Net of California, y Sistemas Médicos Nacionales, S. A., Simnsa, una compañía mexicana (Washington Post 6 de noviembre de 2005). protegen a 25,000 derechohabientes (Kada y Kiy 2004) aunque un año después se reportan 36,000 derechohabientes (Los Ángeles Times 25 de agosto de 2005) con una red de abastecedores de Access Baja para Blue Shield que incluía a 73 médicos en Tijuana, 140 médicos en Baja 
California, 47 farmacias y 3 hospitales. La red Simnsa y Health Net incluye 180 médicos, 15 hospitales y 33 farmacias, en Tijuana, Rosarito, Tecate y Mexicali (Butler 2006).

PRINCIPALES BARRERAS AL INTERCAMBIO DE SERVICIOS DE ATENCIÓN MÉDICA, CUIDADOS A LA SALUD, MEDICAMENTOS Y PRODUCTOS FARMACÉUTICOS

Por otra parte, los analistas hablan de obstáculos. De acuerdo con Osio (2010), quien hace referencia a Schneider, las principales barreras de acceso a los servicios de atención médica y de cuidados de salud son la conveniencia de las distancias, la prolongada espera para cruzar la frontera y lo que denomina como las barreras de no confortabilidad, entre las que se incluyen el temor a proveedores de cuidados de la salud incompetentes y no profesionales, falta de entendimiento de la presencia de proveedores de cuidados de la salud profesionales y de calidad, inconciencia de los ahorros de los costos específicos, el reto de identificar y contactar a un aceptable proveedor de cuidados de la salud, los costos y barreras de idioma de las llamadas de larga distancia para efectuar las citas, y la falta de familiaridad con la localización de las facilidades, las leyes de tráfico y seguridad.

\section{PRINCIPALES CARACTERÍSTICAS DEL INTERCAMBIO DE SERVICIOS DE SALUD, ATENCIÓN MÉDICA, PRODUCTOS FARMACÉUTICOS Y MEDICINAS}

La entrega de servicios de salud en la región de la frontera se caracteriza por un sistema de cuidado y atención médica formado por tres componentes: para residentes con seguro privado y/o ingreso discrecional, con seguro público, y los no asegurados. En San Diego, consumidores de medicamentos y usuarios de servicios médicos sin seguro obtienen gratis y sin compensación cuidado o pagan de su propio bolsillo de acuerdo con una escala de tarifas en las clínicas comunitarias y otros proveedores de seguridad y salud. Los programas de aseguramiento Medical y Medicare están disponibles para segmentos estrictamente definidos de la población y no se encuentran con frecuencia disponibles para muchos trabajadores mexicanos en San Diego. 
Como resultado, muchos trabajadores emigrantes y residentes no califican para la asistencia en cuidados de salud o frecuentemente no tienen acceso a los servicios médicos básicos. En el condado de San Diego, las disparidades étnicas con respecto a la tenencia del seguro médico son más pronunciadas. De los niños, 73\% hispanos tienen seguro médico, comparado con $94 \%$ de africano-americano y $93.4 \%$ de niños blancos (San Diego County 2002: 46) La agencia de Servicios de Salud y Humanos del condado de San Diego, a pesar de que el porcentaje de niños menores de 19 que tienen seguro médico se incrementó en el periodo 1995-2000, 24\% de niños y jóvenes hispanos aún carecen de seguro en el año 2000.

La carencia de seguro médico no significa que la salud de los niños hispanos se descuide por sus padres. El reporte encontró que 88\%, porcentaje más alto de niños hispanos en San Diego, fueron adecuadamente inmunizados, comparado con $79 \%$ de otros grupos étnicos. No obstante, la falta de seguro médico hace muy difícil, si no imposible, para un gran numero de niños hispanos recibir adecuada atención médica, especialmente cuando se requiere atención de especialistas. Los niños y jóvenes de otros grupos étnicos tienen más de 90\% de cobertura (Daily Policy Digest 2002: 26).

La alternativa que tienen es de retornar a la atención médica en Tijuana, la cual es mucho más económica que en San Diego. Hay evidencias científicas de que una porción significante, $41.2 \%$ de la población fronteriza de Estados Unidos, utiliza servicios de salud y cuidados médicos en México. Algunos también retornan a los centros de emergencia en facilidades médicas de San Diego, a pesar de que varios pacientes no pueden pagar sus servicios médicos, menos de 5\% de las visitas a los centros de emergencia, en el condado de San Diego (Orange and San Diego Workgroup 2002).

Mientras que en Tijuana, de acuerdo con datos del Censo 2000, $47.9 \%$ de los residentes tienen cobertura de seguro médico y más de $90 \%$ de esta cobertura se da a través del 
programa de salud pública (Lomelí 2001: 2), dentro de las fronteras de cada país existen comunidades desatendidas o mal servidas.

Algunas áreas de San Diego tienen índices muy altos de pobreza que contradicen su imagen de prosperidad. La tasa de pobreza entre los niños menores de 18 años es de $12 \%$, considerada como baja en un promedio de $24 \%$ para el estado de California. Igualmente, Tijuana, considerada una de las ciudades más ricas de México, tiene muchas colonias populares que carecen de servicios de urbanización básicos, haciéndola vulnerable a las infecciones o enfermedades prevenibles.

La región Tijuana-San Diego, que comparte la frontera de México con Estados Unidos, también comparte muchos aspectos de infraestructura para la atención y servicios de salud, enfermedades infecciosas que cruzan libremente la frontera, tales como tuberculosis, hepatitis, dengue, VIH/SIDA. La alta incidencia de algunas enfermedades entre los hispanos indudablemente que tienen un impacto en la entrega de servicios de salud y en el estatus de residentes de la región fronteriza de estudio.

Tijuana tenía solamente 500 farmacias en 1997, ahora el número es mayor a 1,700. Mientras que en Tijuana hay más de 1,700 farmacias, en San Diego hay aproximadamente 100. Sin embargo, datos más conservadores estiman que en 2002 había aproximadamente 600 farmacias, 1,800 médicos y dentistas en Baja California (Osio 2010). Pero otros datos señalan que en 2007 Tijuana tenía 1,400 farmacias, mientras que San Diego solo contaba con alrededor de 100 (Frontera 30 de abril de2007). Otros estiman que en Tijuana operan 736 farmacias con 3,225 empleados (Inegi 2004), mientras que para el mismo año en el condado de San Diego existían 265 farmacias que empleaban a 5,875 personas (US Census Bureau 2006). En 2002 había aproximadamente 600 farmacias, 1,800 médicos y dentistas en Baja California (Osio 2010). 
El negocio de los farmacéuticos en la frontera de México es una atracción turística y genera cientos de millones de dólares a la economía mexicana. El número de farmacias mexicanas y negocios para ordenar medicinas por correo crecerá más con la legislación que hace el acceso más fácil y el control más difícil.

No existe el equivalente a FDA que revise este creciente número de farmacias en Tijuana. La falta de supervisión médica, la falta de entendimiento del paciente de cómo usar los productos médicos y farmacéuticos correctamente, el indiscriminado uso de los productos, la falta de aseguramiento de calidad y seguridad, son preocupaciones para incrementar la salud de los usuarios. En algunos casos, la venta de medicamentos sobre el mostrador en las farmacias de Tijuana requiere de prescripción médica en Estados Unidos. Muchos residentes en ambos lados de la frontera entre México y Estados Unidos se benefician de medicinas a precios más bajos, sin una consulta médica acreditada, práctica que puede dar lugar a serias consecuencias médicas.

Se puede decir, en general, que este desarrollo tiene dos causas: los altos costos de las medicinas en Estados Unidos y las diferencias de control en las prescripciones y medicinas entre los dos países. En vez de gastar sus ahorros en medicinas y servicios médicos caros, muchos estadounidenses prefieren encontrar otras formas de obtener los mismos servicios a precios asequibles. Para algunos de ellos, viajar a México se ha convertido en una solución viable.

La política existente deja en las manos de los consumidores ejercer su juicio y seguir el consejo de su médico o del farmacéutico; de acuerdo con un maestro de la escuela preparatoria de Nacional City, que nació en Rosarito, quien dice tener seguro médico en Estados Unidos pero que no usa salvo en casos estrictamente necesarios (Escala y Vega 2003). 
Los elevados costos de servicios médicos en Estados Unidos, la pobre cobertura médica de los programas Medicare, las pocas posibilidades para la obtención de programas de seguros médicos para una gran parte de la población, diferencias en los salarios y en el control sobre la prescripciones y medicinas entre los dos países, y la devaluación del peso mexicano, son factores que se relacionan con la alta demanda de los servicios médicos y medicinas mexicanas.

En términos comerciales estrictos, la ventaja que tienen los socios mexicanos frente a sus socios norteamericanos es el bajo costo de los servicios de salud y productos farmacéuticos dentro de sus fronteras y ciertos factores culturales y geográficos que permiten a los proveedores mexicanos acceder al amplio mercado que ofrece a los retirados y pensionados estadounidenses que residen en México, así como la extensiva población hispana que vive en Estados Unidos y Canadá. Warner (s.f.) estima en 80,000 los retirados que viven en Baja California norte que son elegibles para Medicare pero que no les cubre los cuidados de salud fuera de Estados Unidos, lo cual representaría un gran ahorro y por otro lado promovería el desarrollo del sector salud de esta región. Por otro lado, algunos de los desarrollos habitacionales para retirados de Estados Unidos que residen en la región fronteriza mexicana cuentan con servicios hospitalarios y de atención médica (Frontera 22 de octubre de 2007). La salud trasfronteriza incluye temas compartidos por una geografía común y por realidades sociales, demográficas, económicas y culturales de la región Tijuana-San Diego. 


\section{TIPOLOGÍA DE USUARIOS DE LOS SERVICIOS MÉDICOS Y CONSUMIDORES DE} MEDICAMENTOS

Cuatro principales grupos de usuarios de los servicios médicos privados mexicanos y consumidores de medicinas en la frontera de Tijuana se identifican en la actualidad: a) el hispano de origen que habla español, los americanos de origen latino, como los pochos, chicanos y emigrados que viven en Estados Unidos cerca de la frontera; b) estadounidenses, principalmente el segmento de los pensionados y ancianos, y los marginalmente enfermos, usuarios y consumidores; c) tijuanenses usuarios de servicios médicos que consumen medicinas y usan servicios médicos y de salud de Estados Unidos, y d) usuarios y consumidores que desean pólizas para atención médica en ambos lados de la frontera.

\section{EL HISPANO DE ORIGEN QUE HABLA ESPAÑOL, LOS AMERICANOS DE ORIGEN LATINO...}

De los estadounidenses que viajan a México, $87.5 \%$ es para atención médica, $86.7 \%$ para atención dental, y 51.8\% para comprar medicinas (UCLA 2001). No se tienen datos más actualizados.

ESTADOUNIDENSES, PRINCIPALMENTE EL SEGMENTO DE LOS PENSIONADOS Y ANCIANOS... Los consumidores ancianos son impactados por los altos costos de cuidados de la salud ya que tienen que pagar por sus propios productos farmacéuticos, cuidados de enfermería y productos de cuidados de salud (Osio 2010).

\section{TIJUANENSES USUARIOS DE SERVICIOS MÉDICOS...}

De acuerdo con los resultados de un estudio realizado por Guendelman y Jasis (1990), el uso actual de servicios de salud en Estados Unidos por los residentes de Tijuana es bajo. Solo $2.5 \%$ de los residentes cruzan la frontera para recibir atención usualmente de proveedores privados durante un periodo de seis meses, por lo que no necesariamente drenan el sistema de salud pública de Estados Unidos. El perfil sociodemográfico que muestran estos usuarios presenta en su mayor porcentaje características de altos ingresos, mayores de 64 años, con documentos para entrar legalmente, y están asegurados. Guendelman y Jasis (1990) recomiendan estudiar mejor el flujo de las relaciones 
comerciales de servicios de salud y medicinas existentes entre los pacientes de Estados Unidos y los proveedores mexicanos.

USUARIOS Y CONSUMIDORES QUE DESEAN PÓLIZAS PARA ATENCIÓN MEDICA...

No hay estudios concretos para determinar la oferta de este grupo de usuarios y consumidores. Lo que se sabe es que existe una gran cantidad de prospectos que desean la cobertura de servicios médicos mediante los seguros trasfronterizos. Entre estos prospectos se cuentan los migrantes mexicanos y los jubilados y pensionados estadounidenses que residen en México, entre otros grupos.

\section{CUESTIONAMIENTOS PARA FUTURA AVENIDAS DE INVESTIGACIÓN}

Algunos de los principales cuestionamientos en este contexto se relacionan con el impacto de la liberalización comercial en los servicios de salud sobre el costo, calidad y disponibilidad de tales servicios en países de desarrollo, como el caso de México.

¿Cuáles son los tipos de políticas y estrategias adoptadas por otros países para promover las exportaciones de servicios públicos de servicios médicos y qué lecciones pueden desprenderse de estas experiencias?

¿Qué tipos de políticas de apoyo y medidas se requieren para asegurar que el comercio en los servicios de salud no sea a expensas de las prioridades nacionales y de los intereses de los pobres?

Esta investigación deja en claro que el comercio en los servicios de salud lleva a una variedad de preguntas difíciles. Las respuestas a estas cuestiones no pueden generalizarse porque dependen en gran parte de las circunstancias específicas de los países y del ambiente de las políticas. 


\section{CONCLUSIONES E IMPLICACIONES PARA LAS POLÍTICAS PÚBLICAS}

La provisión de servicios de atención médica y cuidados de la salud, así como de medicamentos y otros productos fármacos en Tijuana, y por extensión a la región frontera norte de México, considerada como un destino médico, es una solución significativa para los diferentes segmentos de residentes en California que buscan precios más competitivos. Lo que se reseña en el desarrollo económico de esta metrópoli, Tijuana, es que tiene capacidad para servir a más de 500,000 pacientes mensualmente.

De ahí la necesidad de diseñar e implementar un modelo binacionalmente coordinado en la región Tijuana-San Diego, de cooperación, asistencia y colaboración en un sistema integral de cuidado y atención de servicios médicos y de salud, educación, entrenamiento e investigación y tratamiento de enfermedades y epidemias. Este modelo debe complementar los esfuerzos de US-México Border Health Commission, California Outreach Office, USMBHC-COO, y compartirse con agencias gubernamentales y no gubernamentales para apoyar las iniciativas de amplio alcance.

Ya que se señala como uno de los principales obstáculos que el sector profesional de cuidados de la salud es desorganizado, fragmentado, disperso, no coordinado e inconsistente (Osio 2010), para mejorar la atención y el trato se propone la creación de un mecanismo centralizado que inicie y confirme las citas médicas, despliegue los procedimientos y precios tanto de los diferentes servicios médicos, como de la atención de cuidados de salud, medicinas y otros productos farmacéuticos.

Para ello sería recomendable la integración de una comisión o asociación binacional con funciones de coordinación y colaboración, entre los principales organismos profesionales médicos de ambos lados de la frontera, para desarrollar un acceso rápido a una plaza médica en Tijuana que dé empleo a profesionales de los servicios de cuidados de salud y atención médica certificados, afiliados con los hospitales y clínicas de California, de tal forma que faciliten los mecanismos de citas y de solución al trasporte de los pacientes. 
El sistema de provisión de cuidados de la salud y atención médica de Estados Unidos debe considerar la forma de orientarse culturalmente y en el idioma teniendo en cuenta las necesidades culturales y de lenguaje del paciente. Existe la imperiosa necesidad de mejorar el sistema de provisión de cuidados de la salud y atención médica en Estados Unidos para una mayor competitividad cultural y así atraer al importante sector de latinos que tienen cobertura de seguros médicos, quienes acuden con mayor frecuencia a Tijuana por la necesidad de sentirse culturalmente más competentes y afines porque se sienten más confortables y familiarizados que con la cultura de atención médica en Estados Unidos.

Una forma rápida de paliar esto sería que los hospitales y clínicas de Estados Unidos contrataran a más doctores y enfermeras de origen hispano. Sin embargo, esta situación resulta complicada porque existe escasez de profesionales en el campo de la medicina de origen latino o hispano en el país del norte. Lo que se propone es una política de abrir oportunidades a profesionales de la salud mexicanos para capacitarlos o para trabajar en áreas donde hay una mayor concentración de pacientes latinos. Otra posibilidad es entrenar a futuros profesionales de la salud para ser más culturalmente competentes. También llevar a cabo acuerdos de colaboración con instituciones académicas mexicanas y estadounidenses para la formación de profesionales que realicen prácticas médicas en ambos lados de la frontera sería otra más de las posibles soluciones.

Se propone a todos los miembros del clúster de servicios de salud de Tijuana que adopten sistemas de gestión de relaciones con el cliente —Customer Relationships Management, CRM-, con personal que tenga las habilidades del idioma inglés y con capacidad adecuada para dar atención a prospectos y pacientes que no pueden comunicarse en español. También es importante la instrumentación de un mecanismo de recepción, resolución de quejas, implementación de soluciones y revisión de resultados. 
Así como concretar los esfuerzos de los diversos programas de instituciones y organizaciones binacionales que tienen por objetivo la resolución de los diversos problemas que emergen de las relaciones de intercambio de servicios de atención médica, cuidados de la salud y compra-venta de medicamentos y otros productos farmacéuticos, desde una perspectiva incluyente y para que tengan un impacto en los indicadores de salud, en el crecimiento económico y desarrollo socioeconómico de esta región trasfronteriza.

Es difícil concluir que la relación entre ciudades y pueblos fronterizos sea de dependencia, como es el caso de estudio. De manera general se pueden encontrar relaciones complementarias relacionadas más con la división del trabajo. Sin embargo, uno de los principales puntos que se enfatizan en este trabajo es que sí es posible elevar los ingresos provenientes del comercio de los servicios de salud y mitigar las consecuencias negativas asociadas al diseño de políticas e iniciativas en los ámbitos multilateral, nacional, regional. 


\section{Referencias bibliográficas}

Aguayo, Jennifer, E. Richard Brown, Michael A. Rodríguez and Lia Margolis, 2003, «Important Health Care Issues for California Latinos: Health Insurance and Health Status». Health Policy Fact Sheet. In http://www.healthpolicy.ucla.edu/pubs/publication.asp? pubID=60\#download [accessed: 15/1//04].

American Cancer Society, 1991-2002, Actualización de American Cancer Society 1991. «Questionable Cancer Practices in Tijuana and Other Mexican Border Clinics». Cancer Journal Clinic, n. 41, pp. 310-319. En http://documentscancer.org/232.001 [consulta: 16 de marzo de 2002].

2007, Medical Travel Outside the US. 10 pp.

Anfuso, Dawn, 1995, «HR's role in creating Mexico’s first HMO». Personnel Journal, v. 74, n. 5 , pp. 59-76.

Brown, E. Richard, Ninez Ponce, Thomas Rice and Shana Alex Lavarreda, 2003, The State of Health Insurance in California: long-term and intermittent lack of health insurance coverage. UCLA, Center for Health Policy Research. In http://www.healthpolicy.ucla.edu/ pubs/publication.asp?pubID=78 [accessed: 1/14/04].

Butler, K. M., 2006, «Cross-Border HMOS Improve Cost Savings, Cultural Sensitivities». Employee Benefit News. February.

California HealthCare Foundation, 2001, Prescription Drug Use and Expenditures in California: Key Trends and Drivers. California HealthCare Foundation, California.

COBBH, 2000, Annual Border Health Status Report. California Office of Binational Border Health, California. 
Daily Policy Digest, 2002, «County of San Diego Health and Human Services Agency». Report Card 2001, Daily Policy Digest, April 29.

Darcé, Keith, 2008, «Insurer's lower-cost plans offer Latinos care in U.S., Mexico». UnionTribune, February 28.

Doheny, K., 2006, «El turismo médico levanta vuelo». HealthDay News/Hispanizare, 6 de julio.

El Mexicano, «Gasta turismo de salud 2 mil mdd». 11 de junio de 2007.

Escala Rabadán, Luis and Vega German, 2003, «Cross-Border Commuters in the Tijuana San Diego Region: Insights from Case Studies». Presented at Center for U.S.MexicaStudies. University of California, San Diego, November 14.

Escobedo, L. y V. M. Cardenas, 2006, «Utilization and Purchase of Medical Care Services in Mexico by Residents in the United States of America, 1998-1999». Revista Panamericana de Salud Pública Panamerican Journal of Public Health, v. 19, n. 5, pp. 300-305.

Families USA Foundation, 1992, Crossing to Mexico: Priced out of American Health Care. Washington, D. C.

Frontera, 2006, «Eligen a Tijuana pacientes extranjeros». 13 de junio, p. 1.

2007, «Ahogan sin remedio farmacias a Tijuana». 30 de abril, p. 1.

2007, «Dejan turistas unos 2 mil mdd». 17 de junio, p. 14. 
2007, «Inicia esta semana congreso dental». 22 de octubre, p. 23.

Guendelman, S. and M. Jasis, 1990, «Measuring Tijuana Residents' Choice of Mexican or U.S. Health Care Services». Public Health Reports, v. 195, n. 6, pp: 575-583, NovemberDecember.

Guendelman, S. and T. H. Wagner, 2000, «Health Services Utilization among Latinos and White non-Latinos: Result form a National Survey». Journal of Health Care for the Poor and Underserved, v. 11, n. 2, pp. 189.

Inegi, 2004, Censos Económicos.

International Community Foundation, 2008a, «Issues, impacts and solutions: health». In International Community Foundation, Blurr Borders. In http://www.icfdn.org [consulta: 8 de abril de 2010].

2008b, «Health and human services: Building healthy binational communities through cross-border cooperation». In Chapter VII, International Community Foundation, Blurr Borders. In http://www.icfdn.org [consulta: 8 de abril de 2010].

Kada N. y R. Kiy (eds.), 2004, Blurred Borders: Trans-Boundary Impacts \& Solutions in the San Diego-Tijuana Border Region, s./l. International Community Foundation. San Diego, CA.

Lomelí, Blanca, 2001, «La Salud en Baja California: Necesidades y Oportunidades desde Una Perspectiva Comunitaria». Mimeo.

Los Angeles Times, 2005, «Healthcare Is Migrating South of the Border». 25 de agosto. 
Macias, E. P. y L. S. Morales, 2001, «Crossing the Border for Health Care». Journal of Health Care for the Poor and Underserved, v. 12, n. 1, pp. 77-87.

Moss, R. W., 2005, «Patient Perspectives: Tijuana Cancer Clinics in the Post-nafta era». Integrative Cancer Therapies, v. 4, n. 1, pp. 65- 86.

National Latino Research Center, 2004, «The Border that Divides and Unites: Addressing Border Health in California». October 2004 Research Report Prepared for The California Endowment by the NATIONAL LATINO RESEARCH CENTER, California State, University San Marcos.

Olivieri, F., 2007, «An Rx From Ancient Times. Baja sees economic opportunity in promoting health tourism». San Diego Metropolitan, September.

Orange and San Diego Workgroup, 2002. «Insure the Uninsured Project. Charts». In http://www.work-and-health.org/regionalWG/OrangeSDCounty/OrangeandSD.html [accessed: 3/8/04].

Osio, Sal, 2010, «Tijuana: The medical destination». Sign up and win a DVD of the documentary: The North Baja coast. Transborder Communications.

Ramírez, Miguel Ángel, 2008, «Turismo médico en Tijuana, México». Escuela de negocios. CESUN UNIVERSIDAD, Tijuana, México.

Robert Wood Johnson Foundation, 2005, «Characteristics of the Uninsured: A View from the States». Prepared for the State Health Access Data Assistance Center, University of Minnesota. Using data from the Center for Disease Control and Prevention's 2003 Behavioral Risk Factor Surveillance System Survey (brfss). 
San Diego Dialogue, 1999, «Who Crosses the Border». June.

San Diego County, 2002, San Diego County Child and Family Health and WellBeingReport Card 2000. San Diego County, San Diego.

San Diego Union Tribune, 2005, «Cross-border Health Insurance is a Hit with Employers and Workers». October 16.

Secretaría de Turismo de Baja California y Comité de Turismo y Convenciones de Tijuana, 2003, Impacto del sector turismo en la economía de Baja California. Mexicali, Tijuana.

Sharp, John, 1998, Bordering the future: challenge and opportunity in the Texas border region. Texas Comptroller of Public Accounts, Austin, Texas.

Smith, R. L., 1968, «New Traffic in "Cures for Cancer"». Saturday Evening Post, v. 241, n. 3, pp. 62-64.

Terman, Elias, 2004, «From TVs To Biomedical Devices». San Diego Metropolitan. December

Tijuana DMA Estimates, 2002, «Tijuana, Tecate \& Rosarito». Crossborder Business Associates, n. 619, pp. 710-812.

UCLA, 2001, California Health Interview Survey (CHIPS). In http://www.chis.ucla.edu.

UNCTAD/WHO, 1998, Regional trade between the US and Mexico in the context of NAFTA, Chapter 10. Unctad/Who, Washington.

US Census Bureau, 2006, California 2004. County Business Patterns, CBP/04-6. 
US Senate Special Commite on Aging, 2006, The Globalization of Health Care: Can Medical Tourism. US Senate Special Commite on Aging, USA.

Valdez, R. Burciaga, Hal Morgenstern, E. Richard Brown, Roberta Wyn, Chao Wang and William Cumberland, 1993, «Insuring Latinos Against the Costs of Illness». Journal of the American Medical Association, 2, v. 69, n. 7, pp. 889-94, February 17.

Villa-Caballero, Leonel, Víctor Manuel Caballero-Solano, y Olga Alicia Andrade-Barreto, 2008, «Globalización y salud en la región San Diego-Tijuana». Gaceta Médica Mexicana, v. 144 , n. 5 , pp. 389-394.

Wallace, Steven P. and Ybanez, Elmyra, 2006, «Trans-border relations and health care in Tijuana». UC Mexus Collaborative Grants. June 26.

Warner, David C., s.f., «Health and Medical Care in San Diego and Tijuana: Prospects for Collaboration», San Diego Dialogue. http://www.sandiegodialogue.org/pdfs/health\% 20exec\%20summ\%2012-10.pdf [consulta: 7 de abril de 2010].

Washington Post, 2005, «Passport to Health Care at Lower Cost to Patient». 6 de noviembre, p. 3.

Fecha de recepción: 18 de enero de 2010.

Fecha de aceptación: 12 de abril de 2010. 